\title{
Prototheca stagnora
}

National Cancer Institute

\section{Source}

National Cancer Institute. Prototheca stagnora. NCI Thesaurus. Code C124362.

A species of achlorophyllic alga in the family Chlorellaceae. P. stagnora is mucoid and grows only at 30 degrees $C$. 